\title{
VARIABLE SAMPLING PLAN BASED ON CPMK INVOLVING MINIMUM SUM OF RISK
}

\section{S. GEETHA}

Department of Statistics, PSG College of Arts \& Science, Coimbatore, Tamil Nadu, India

\begin{abstract}
Process capability indices are useful management tools to improve the quality of manufacturing processes and the quality inspection of products. Process capability indices provides common quantitative measures on manufacturing capability and production quality. Acceptance sampling is a classification of SQC and consists of procedures for making a decision about one or more lots of finished items or materials produced by a manufacturing process. Sampling inspection by variables is one among the various classifications of acceptance sampling and consists in a procedure for deciding about the disposition of a lot of individual units by using sample measurements of units on a quality characteristic under study. Any sampling plan involves two types of risks, producer's risk and Consumer's risk. It is not possible to minimize both the risks simultaneously; However it is possible to minimize the sum of these risks. In this paper a new variables sampling plan based on process capability index $C_{p m k}$ involving minimum sum of producer's and consumer's risk for specified $C_{A Q L}$ and $C_{L T P D}$ is introduced. The decisions made are more accurate and reliable since the proposed sampling plan is based on the exact sampling distribution. The required sample sizes and the corresponding critical acceptance values for various producer's risk, the consumer's risk and the capability requirements, acceptance quality level (AQL), and the lot tolerance percent defective (LTPD) are provided. A case study is also presented to illustrate how the proposed procedure can be constructed and applied to the real applications.
\end{abstract}

KEYWORDS: Acceptance Sampling Plans, Critical Acceptance Values, Process Capability Analysis \& Minimum Sum of Risk

Received: Dec 01, 2018; Accepted: Dec 20, 2018; Published: Feb 04, 2019; Paper Id.: IJMCARJUN20195

\section{INTRODUCTION}

One of the important aspects of quality insurance is the inspection of raw materials, Semi finished products, and finished products. Acceptance sampling is the type of inspection procedure employed to acceptance or rejection of products based on adherence to a standard. Acceptance sampling plans consists of various inspection procedure called sampling plans, which are used for drawing inferences about one or more lots of finished products based on the results of the inspection of one or more random samples drawn from the lot(s). There are a number of different ways to classify acceptance sampling plans. One major classification is by attributes and variables. When a quality characteristic is measurable on a continuous scale and is known to have a distribution of a specified type, it may be possible to use as a substitute for an attributes sampling plan based on sample measurements such as the mean and the standard deviation of the sample. A variables sampling is advantageous in the sense that it generates more information from each item inspected, requires small sample and provides same protection when compared to attributes sampling. [See, Bowker and Goode (1952), and 
Montgomery (2004)]. On the basis of the implicit assumption that the quality characteristic is distributed according to normal with mean $\mu$ and standard deviation $\sigma$, the concept of variables sampling inspection has been studied by many researchers. Some of the early works on variables sampling inspection are seen in Lieberman and Resnik off (1955), Schilling (1982), Owen (1966, 1967), and Hamaker (1979). Studies relating to sampling plans when the assumption of normality of the quality characteristic fails or the functional form of the underlying distribution deviates from normal or the form of the distribution is not known are also found in the literature of acceptance sampling. Duncan (1986), Srivastava (1961), Das and Mitra (1964), Owen (1969), Takagi (1972), Guenther (1972, 1985), Zimmer and Burr (1963), Aminzadeh (1996), Suresh and Ramanathan (1997), Vijayaraghavan and Geetha (2010,2011b,2013) are a few references which deal with variables inspection using non-normal distributions.

In a sampling plans there is no guarantee that every defective item in a lot is inspected, the sampling involves risks of not adequately reflecting the quality conditions of the lot. Such risk is even more significant as the rapid advancement of the manufacturing technology and stringent customers demand is enforced. Particularly, when the fraction of nonconforming products is required very low, such as measured in parts per million (PPM), the required number of inspection items must be very large in order to adequately reflect the actual lot quality. As today's modern quality improvement philosophy, reduction of variation from the target value is the guiding principle as well as reducing the fraction of nonconformities. Process Capability Indices has become widely adopted as the means of Perfomance to evaluate the ability of a process to satisfy customer requirements in terms of specification limits. Several authors have promoted the use of various Process Capability Indices and examined with different degrees of completeness (see Pearn et al., 1992, 1998; Kotz and Lovelace, 1998; Kotz and Johnson, 2002; Spiring et al., 2003). Negrin (2009, 2011), Aslamet al. (2013), Aslam et al. (2014)Aslam et al. (2015a); Yen et al.(2015); Aslam et al. (2015b);. are references deals with different sampling plans using capability indices.

The operating characteristic (OC) curve is used as a criterion for measuring the performance of an acceptance sampling plan which quantifies for the producers risk and consumers risk.

The OC curve plots the probability of accepting the lot versus the lot fraction nonconforming, which displays the discriminatory power of the sampling plan. For product quality protection and the company's profit, both the producer and the consumer would focus on certain points on the OC curve to reflect their benchmarking risk and hence one allows certain levels of risks for producer and consumer Unfortunately, it is not possible to simultaneously minimize the producer's and consumer's risks without letting $n$ get unreasonably large. However, it is possible to minimize the sum of producer's and consumer's risks. Golub (1953) has introduced the method of minimizing the sum of the producer's and consumer's risks for acceptance sampling plan by the attributes for the fixed sample size for specified AQL and LTPD. Soundarajan (1981) has been extended the Golub approach for single sampling plan by assuming a Poisson model. Govidaraju(1984) has given the table for the selection of single sampling plan which minimizes the sum of produces and consumer's risks without specifying the sample size under Poisson model. Soundarajan \& Vijayaraghavan (1989) has studied multiple deferred state sampling plan (MDS-1 $(0,2)$ ) involving a minimum sum of risk. Govindaraju and Subramani (1990) have given the procedure for the selection of single sampling plans of attributes involving a minimum sum of risk without fixing the sample size under Poisson model. Govindaraju and Subramani (1992) have used minimum sum of risk for various sampling plans. Subramani (2004) have given a review of minimum sum of risk. V.Kaviyarasu, G. Sangamesh waran (2013) have studied the QSS deferred sampling using the minimum sum of risk. D. Senthilkumaret. Al 
(2014) has studied Multiple deferred state(MDS-1) plan involving a minimum sum of risk.

The capability index $\mathrm{C}_{\mathrm{pmk}}$ is constructed by combining the yield-based index $\mathrm{C}_{\mathrm{pk}}$ and the loss-based index $\mathrm{C}_{\mathrm{pm}}$, taking into account the process yield (meeting the manufacturing specifications) as well as the process loss (variation from the target). Wu et.al (2008) has studied the variable sampling plan based on $\mathrm{C}_{\mathrm{pmk}}$ for product acceptance determination. In this paper the variable sampling plan based on $\mathrm{C}_{\mathrm{pmk}}$ involving a minimum sum of producer's risk and consumer's risk is proposed.

\section{PROCESS CAPABILITY INDICES}

Process Capability Indices were defined as the quantitative indicators relate the performance of process or product characteristic to the specifications. The different probability indices have been defined explicitly as below, where $\mu$ is the process mean, $\sigma$ is the process standard deviation, $\mathrm{U}$ and $\mathrm{L}$ are the upper and lower specification limits, $\mathrm{T}$ is the target value preset by customers or product designers, $d=\frac{U-L}{2}$ is half of the length of the specification interval:

$$
C_{p}=\frac{(U-L)}{6 \sigma}, C_{p k}=\min \left(\frac{(\mu-L)}{3 \sigma}, \frac{(U-\mu)}{3 \sigma}\right), C_{p m}=\frac{(U-L)}{6 \sqrt{\sigma^{2}+(\mu-T)^{2}}} C_{p m k}=\min \left(\frac{(\mu-L)}{3 \sqrt{\sigma^{2}+(\mu-T)^{2}}}, \frac{(U-\mu)}{3 \sqrt{\sigma^{2}+(\mu-T)^{2}}}\right),
$$

$C_{p}$ is the Process potential index reflects consistency of product quality characteristics. $C_{p k}$ takes into account the magnitude of process variance as well as the departures of process mean from the mid-point of specification limits. $C_{p m}$ is the Taguchi index distinct as the capacity of the process to be grouped around the target or nominal value, which is the dimension that meets to exact anticipated value for the quality characteristic. $C_{p m k}$ is an hybrid index derived from $C_{p k}$ and $C_{p m}$ which increases the sensitivity to departures of the process mean $\mu$ from the desired target value $\mathrm{T}$ where $\mathrm{T}=\mathrm{M}=\frac{U+L}{2}$. For capability measure due to high standard and stringent requirement on product quality and reliability $C_{p m k}$ will be an appropriate measure.

\section{ESTIMATION OF $C_{P M K}$ AND ITS SAMPLING DISTRIBUTION}

When the process distributed as normal that is the process is stable (under statistical control), Pearn et al.(1992) suggested using the natural estimator which is defined as

$$
\hat{C}_{p m k}=\min \left\{\frac{U-\bar{X}}{3 \sqrt{S_{n}^{2}+(\bar{X}-T)^{2}}}, \frac{\bar{X}-L}{3 \sqrt{S_{n}^{2}+(\bar{X}-T)^{2}}}\right\}=\frac{d-|\bar{X}-M|}{3 \sqrt{S_{n}^{2}+(\bar{X}-T)^{2}}} \text { Where } \bar{x}=\sum_{i=1}^{n} \frac{x_{i}}{n} \text { and } S_{n}^{2}=\sum_{i=1}^{n} \frac{\left(X_{i}-\bar{x}\right)^{2}}{n} \text { are the MLE of } \mu \text { and } \sigma^{2}
$$

respectively. $\quad S_{n}^{2}+(\bar{x}-T)^{2}$ is the uniformly minimum variance unbiased estimator of $\sigma^{2}+(\mu-T)^{2} . \quad \hat{C}_{p m k}$ is the estimator of $C_{p m k}$, which is given by $\hat{C}_{p m k}=\frac{D-\sqrt{H}}{3 \sqrt{K+H}}$ where $D=\frac{d \sqrt{n}}{\sigma}, K=\frac{n S_{n}^{2}}{\sigma^{2}}, H=\frac{n(\bar{x}-T)^{2}}{\sigma^{2}}$ and $\eta=\frac{\sqrt{n}|\mu-T|}{\sigma}$. When the process is normally distributed $K$ is distributed as a chi square distribution with $n-1$ degrees of freedom. $H$ is distributed as a non central chi-square distribution with one degrees of freedom with non centrality parameter $\lambda=\frac{n(\mu-T)^{2}}{\sigma^{2}} . \sqrt{H}$ is distributed as the normal distribution with mean $\eta$ and variance 1 . The estimator $\hat{C}_{p m k}$ is a mixture of the chi-square distribution and the 
non-central chi-square distribution, as expressed in the following.

$$
\hat{C}_{p m k} \approx \frac{\frac{d \sqrt{n}}{\sigma}-\chi_{1, \lambda}^{\prime}}{3 \sqrt{\chi_{n-1}^{2}+\chi_{1, \lambda}^{\prime 2}}}
$$

Chen and Hsu (1995) investigated the asymptotic sampling distribution of the estimated $C_{p m k}$ and showed that the estimator $\hat{C}_{p m k}$ is consistent, asymptotically unbiased estimator of $C_{p m k}$, and if the fourth moment of the distribution of $\mathrm{X}$ is finite, then $\hat{C}_{p m k}$ is asymptotically normal. Vannman and Kotz (1995) obtained the distribution of the estimated $\mathrm{C}_{\mathrm{p}}(\mathrm{u}, \mathrm{v})$ for cases with $\mathrm{T}=\mathrm{M}$. By taking $\mathrm{u}=1$ and $\mathrm{v}=1$, the distribution of $\mathrm{C}_{\mathrm{p}}(\mathrm{u}, \mathrm{v})=C_{p m k}$ can be obtained. Wright (1998) derived an explicit but rather complicated expression for the probability density function of the estimated $C_{p m k}$. Using variables transformation and the integration technique similar to that presented in Vannman (1997), the cumulative distribution function (CDF) and the probability density function (PDF) of the estimated index $\hat{C}_{p m k}$ may be expressed alternatively in terms of a mixture of the chi-square distribution and the normal distribution. The explicit form of the CDF considerably simplify the complexity for analysing the statistical properties of the estimated index, which can be expressed below (Pearn and Lin(2002)

$$
F_{\hat{C}_{p m k}}(x)=1-\int_{0}^{b \sqrt{n} /(1+3 x)} G\left(\frac{(b \sqrt{n-t})^{2}}{9 x^{2}}-t^{2}\right) \times[\varphi(t-z \sqrt{n})+\varphi(t+z \sqrt{n})] d t
$$

for $\mathrm{x}>0$, where $\mathrm{b}=\mathrm{d} / \sigma, z=(\mu-T) / \sigma, \mathrm{G}($.$) is the CDF of the chi-square distribution, \chi_{n-1}^{2}$, with $n-1$ degrees of freedom, $\varphi($.$) is the PDF of the standard normal distribution \mathrm{N}(0,1)$ and it is noted that we would obtain an identical equation if we substitute $\mathrm{z}$ by $-\mathrm{z}$ in above equation for fixed values of $\mathrm{x}$ and $\mathrm{n}$. Noting that for $\mu>\mathrm{U}$ or $\mu<\mathrm{L}$, the capability $\mathrm{C}_{\mathrm{pmk}}<0$, and for $\mu=\mathrm{U}$ or $\mu=\mathrm{L}$, the capability $\mathrm{C}_{\mathrm{pmk}}=0$. The requirement with $\mathrm{L}<\mu<\mathrm{U}$ has been a minimum capability requirement applies to most start-up engineering applications or new processes.

\section{SINGLE SAMPLING PLANS BY VARIABLES BASED ON C $_{\text {PMK INVOLVING MINIMUM SUM OF RISKS }}$}

When the individual quality characteristic of interest is measurable on a continuous scale and is normally distributed with mean $\mu$ and standard deviation $\sigma$, the $\mathrm{U}$ and the $\mathrm{L}$ can be defined as the acceptable values of this parameter. A variables sampling plan is designed with a specified OC curve. Let $\left(C_{A Q L}, 1-\alpha\right)$ and $\left(C_{L T P D}, \beta\right)$ be the two points on the OC curve of interest, where $C_{A Q L}$ and $C_{L T P D}$ represent the capability requirements corresponding to the AQL and the LTPD based on $\mathrm{C}_{\mathrm{pmk}}$ index, respectively. The sampling plan is given by

If $C_{p m k} \geq \mathrm{C}_{\mathrm{AQL}}$, then the lot should be accepted with producer's risk $\alpha$, and

If $C_{p m k} \leq \mathrm{C}_{\mathrm{LTPD}}$, then the lot should be rejected with consumer's risk $\beta$,

As described previously, owing to the sampling distribution of $\hat{C}_{p m k}$ is expressed in terms of a mixture of the 
chi-square and the normal distributions. For processes with $\mathrm{T}=\mathrm{M}$, the index may be rewritten as $C_{p m k}=(d / \sigma-|z|) /\left[3\left(1+z^{2}\right)^{1 / 2}\right]$, where $z=(\mu-T) / \sigma$. Further, given $C_{p m k}=C, b=d / \sigma$ can be rewritten as $b=3 C\left(1+z^{2}\right)^{1 / 2}+|z|$ The probability of accepting the lot can be expressed as

$$
P_{a}\left(C_{p m k}\right)=P\left(\hat{C}_{p m k} \geq C_{m} \mid C_{p m k}=C\right)=\int_{0}^{b \sqrt{n} /\left(1+3 C_{m}\right)} G\left(\frac{(b \sqrt{n-t})^{2}}{9 C_{m}{ }^{2}}-t^{2}\right) \times[\varphi(t-z \sqrt{n})+\varphi(t+z \sqrt{n})] d t
$$

The expression for the sum of Producer's and Consumer's risk is given by

$$
\alpha+\beta=1-P_{a}\left(C_{A Q L}\right)+P_{a}\left(C_{L T P D}\right)
$$

The required inspection sample size $n$ and critical acceptance value $C_{m}$ of $\hat{C}_{p m k}$ for the proposed sampling plans is obtained by solving the following conditions with simultaneously minimizing sum of producer's and Consumer's risk.

$$
\begin{aligned}
& 1-\alpha \leq \int_{0}^{b_{1} \sqrt{n} /\left(1+3 C_{m}\right)} G\left(\frac{\left(b_{1} \sqrt{n-t}\right)^{2}}{9 C_{m}{ }^{2}}-t^{2}\right) \times[\varphi(t-z \sqrt{n})+\varphi(t+z \sqrt{n})] d t \\
& \beta \geq \int_{0}^{b_{2} \sqrt{n} /\left(1+3 C_{m}\right)} G\left(\frac{\left(b_{2} \sqrt{n-t}\right)^{2}}{9 C_{m}{ }^{2}}-t^{2}\right) \times[\varphi(t-z \sqrt{n})+\varphi(t+z \sqrt{n})] d t
\end{aligned}
$$

Where $b_{1}=3 C_{A Q L}\left(1+z^{2}\right)^{1 / 2}+|z|$ and $b_{2}=3 C_{L T P D}\left(1+z^{2}\right)^{1 / 2}+|z|, C_{A Q L}>C_{L T P D}$.

For practical applications purpose, the critical acceptance values and the sample sizes required for the sampling plans are calculated and tabulated with commonly used $\alpha, \beta, C_{A Q L}$ and $C_{L T P D}$ using MATLAB Program. Table 1 displays $n$ and $C_{m}$ for $\alpha=0.01,0.025(0.025) 0.1, \quad \beta=0.01,0.025(0.025) \quad 0.1$, with various benchmarking quality levels $\left(C_{A Q L}, C_{L T P D}\right)=(1.33,1.00),(1.5,1.00),(1.5,1.33),(1.67,1.33)$. For example if the quality level $\left(C_{A Q L}, C_{L T P D}\right)$ set to $(1.5,1.00)$ the producers risk is 0.05 and the consumer risk is 0.1 the corresponding sample size and critical acceptance value can be calculated as $\left(\mathbf{n}, \boldsymbol{C}_{\boldsymbol{m}}\right)=(\mathbf{4 2 , 1 . 2 2 2 6})$ which gives minimum riskm $=\mathbf{0 . 1 3 0 6}$. It shows that the lot will be accepted if the 42 inspected items yield measurement with $\hat{C}_{p m k} \geq 1.2226$. Otherwise we do not have sufficient information to conclude that the process meet the present capability requirement. In this case we would believe that $C_{p m k} \leq C_{L T P D}$. So the consumer will reject the lot. 
Table 1: $\left(\mathrm{n}, C_{m}\right)$ Values for $\alpha$ Risk $=0.01,0.025(0.025) 0.1, \beta$-Risk $=0.01$, $0.025(0.025) 0.1$ with Various $\left(C_{A Q L}\right.$ and $\left.C_{L T P D}\right)$

\begin{tabular}{|c|c|c|c|c|c|c|c|c|c|c|c|c|c|}
\hline \multirow[t]{2}{*}{$x$} & \multirow[t]{2}{*}{$\boldsymbol{\beta}$} & \multicolumn{3}{|c|}{$\mathrm{C}_{\mathrm{AQL}}=1.33, \mathrm{C}_{\mathrm{LTPD}}=1.00$} & \multicolumn{3}{|c|}{$\mathrm{C}_{\mathrm{AQL}}=1.50, \mathrm{C}_{\mathrm{LTPD}}=1.00$} & \multicolumn{3}{|c|}{$\begin{array}{l}\mathrm{C}_{\mathrm{AQL}}=1.50 \\
\mathrm{C}_{\mathrm{LTPD}}=1.33\end{array}$} & \multicolumn{3}{|c|}{$\begin{aligned} \mathrm{C}_{\mathrm{AQL}} & =1.67, \mathrm{C}_{\mathrm{LTPD}} \\
& =1.33\end{aligned}$} \\
\hline & & $n$ & $C_{m}$ & $m$ & $n$ & $C_{m}$ & $m$ & $n$ & $C_{m}$ & $m$ & $n$ & $C_{m}$ & $m$ \\
\hline \multirow{5}{*}{0.010} & 0.010 & 205 & .1630 & 0.019 & 102 & 1.2469 & 0.0174 & 1043 & 1.4145 & 0.0197 & 287 & 1.4986 & 0.0196 \\
\hline & 0.025 & 175 & 1499 & 0.03 & 87 & 2264 & 0.0295 & 878 & 1.4076 & 0.0348 & 247 & 1.4848 & 0.0323 \\
\hline & 0.050 & 150 & 1.1364 & 0.0547 & 75 & 1.2059 & 0.0504 & 753 & 1.4005 & 0.059 & 207 & 1.4708 & 0.0578 \\
\hline & 0.075 & 135 & 1.1265 & 0.07 & 65 & 1.19 & 0.0769 & 672 & 1.3955 & 0.08 & 186 & 1.4602 & 0.0817 \\
\hline & 0.100 & 125 & 1.1180 & 0.0992 & 58 & 1.17 & 0.10 & 616 & 1.3507 & 0.1087 & 171 & 1.4514 & 0.1051 \\
\hline \multirow{5}{*}{0.025} & 0.010 & 177 & 1.1777 & 0.03 & 88 & 1.2686 & 0.0314 & 889 & 1.4217 & 0.0344 & 248 & 1.5135 & 0.0337 \\
\hline & 0.025 & 147 & 1.1640 & 0.0471 & 73 & 1.2482 & 0.0445 & 742 & 1.4150 & 0.0493 & 208 & 1.4992 & 0.0470 \\
\hline & 0.050 & 123 & 1.1504 & 0.0713 & 63 & 1.2282 & 0.0632 & 626 & 1.4079 & 0.0734 & 174 & 1.4855 & 0.0713 \\
\hline & 0.075 & 112 & 1.1404 & 0.0906 & 52 & 1.2126 & 0.0965 & 555 & 1.4025 & 0.0978 & 154 & 1.4748 & 0.0953 \\
\hline & 0.100 & 100 & 315 & 0 & 4 & 1.1997 & 0.11 & 505 & 1.3975 & 0.1225 & 139 & 1.4656 & 0.1201 \\
\hline \multirow{5}{*}{0.050} & 0.010 & 154 & 1.1923 & 0.0571 & 77 & 1.2911 & 0.0550 & 766 & 1.4293 & 0.0595 & 216 & 1.5285 & 0.0577 \\
\hline & 0.0 & 128 & 39 & & 6 & 1.2710 & 0.0 & 631 & 1.4222 & 0.0739 & 178 & 1.5147 & 0.0711 \\
\hline & 0.050 & 106 & 1.1652 & 0.0921 & 54 & 1.2508 & 0.0845 & 523 & 1.4150 & 0.0988 & 147 & 1.5002 & 0.0952 \\
\hline & 0.075 & 93 & 555 & 150 & 46 & 1.2358 & 0.1112 & 458 & 1.4100 & 0.1235 & 128 & 1.4901 & 0.1200 \\
\hline & 0.100 & 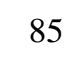 & 1465 & 1350 & 42 & .2226 & 0.1306 & 413 & 1.4053 & 0.1472 & 116 & 1.4807 & 0.1422 \\
\hline \multirow{5}{*}{0.075} & 0.010 & 139 & 1.2033 & 0.0823 & 72 & 1.3075 & 0.0756 & 693 & 1.4348 & 0.084 & 196 & 1.5397 & 0.0821 \\
\hline & 0.025 & 116 & 1.1903 & 0.0918 & 57 & 1.2884 & 0.0913 & 564 & 1.4279 & 0.0984 & 158 & 1.5262 & 0.0970 \\
\hline & 0.050 & 92 & 1.1768 & 0.1203 & 47 & 1.2682 & 0.1119 & 461 & 1.4207 & 0.1238 & 129 & 1.5122 & 0.1214 \\
\hline & 0.075 & 81 & 1.1665 & 0.1412 & 41 & 1.2531 & 0.1334 & 401 & 1.4152 & 0.1485 & 113 & 1.5015 & 0.1437 \\
\hline & 0.100 & 73 & 1.1576 & 0.1624 & 38 & 1.2402 & 0.1478 & 358 & 1.4109 & 0.1727 & 102 & 1.4923 & 0.1648 \\
\hline \multirow{5}{*}{0.100} & 0.010 & 129 & 1.2125 & 0.1064 & 66 & 1.3222 & 0.1018 & 639 & 1.4395 & 0.1087 & 181 & 1.5492 & 0.1069 \\
\hline & 0.025 & 104 & 1.2000 & 0.1197 & 55 & 1.3032 & 0.1081 & 513 & 1.4327 & 0.1236 & 146 & 1.5362 & 0.1203 \\
\hline & 0.050 & 75 & 1.1865 & 0.1659 & 44 & 1.2840 & 0.1313 & 419 & 1.4258 & 0.1476 & 118 & 1.5225 & 0.1445 \\
\hline & 0.075 & 75 & 1.1765 & 0.1599 & 37 & 1.2692 & 0.1580 & 359 & 1.4202 & 0.1735 & 101 & 1.5121 & 0.1698 \\
\hline & 0.100 & 65 & .1678 & 0.1879 & 34 & 1.2562 & 0.1724 & 319 & 1.4155 & 0.1975 & 89 & 1.5028 & 0.1947 \\
\hline
\end{tabular}

\section{Example}

A case study on Load Cell manufacturing process is presented to show how the sampling plan can be established and applied. Load cell is popularly used in making the Weighing devices, Weighing Gauge, Elevators, etc. The factory manufactures various types of the Load cells. For a particular model of the Load cell investigated, the target sensitivity 
value is set to $1.5 \mathrm{mv} / \mathrm{V}$, and the tolerance of sensitivity is $0.15 \mathrm{mv} / \mathrm{V}$, that is, the USL of the load cell's sensitivity is 1.65 $\mathrm{mv} / \mathrm{V}$, the LSL of the load cell's sensitivity is $1.35 \mathrm{mv} / \mathrm{V}$. If the product characteristic does not fall within the tolerance (LSL, USL), the lifetime or reliability of the load cell will be discounted. In the contract, the $\mathrm{C}_{\mathrm{AQL}}$ and $\mathrm{C}_{\mathrm{LTPD}}$ are set to 1.67 and 1.33 based on $\mathrm{C}_{\mathrm{pmk}}$ index with both producers's and Consumers's risk is 0.1 . Therefore, the problem for quality practitioners is to determine the critical acceptance value and the required sample size of the sampling plan that provide the desired levels of protection for both the producer and the consumer with minimum sum of risks. Based on the proposed procedure, we can obtain the critical acceptance value and inspected sample size are $\left(n, C_{m}\right)=(89,1.5028)$ with minimum risk $\mathrm{m}=0.1946$. Hence, the samples are simulated using software and given in the table 2 , we obtain that

$$
\bar{X}=1.513, \quad S_{n}=0.026, \quad \hat{C}_{p m k}=\left\{\frac{U S L-\bar{X}}{3 \sqrt{S_{n}^{2}+(\bar{X}-T)^{2}}}, \frac{\bar{X}-L S L}{3 \sqrt{S_{n}^{2}+(\bar{X}-T)^{2}}}\right\}=1.5709
$$

Therefore, in this case, the consumer would accept the entire lot, since the sample estimator from the inspections, 1.5709 , is greater than the acceptance value 1.5028 of the sampling plan.

Table 2: (Sample Data with 89 Observations)

\begin{tabular}{|l|l|l|l|l|l|l|l|l|l|}
\hline 0.8353 & 0.5483 & 0.6279 & 0.6357 & 0.6482 & 0.5870 & 0.6288 & 0.7512 & 0.6793 & 0.6916 \\
\hline 0.7524 & 0.6269 & 0.6928 & 0.8356 & 0.5781 & 0.7214 & 0.6699 & 0.6415 & 0.7014 & 0.8799 \\
\hline 0.7527 & 0.7577 & 0.5501 & 0.7777 & 0.6441 & 0.6690 & 0.6834 & 0.6412 & 0.6519 & 0.6182 \\
\hline 0.6894 & 0.6677 & 0.8524 & 0.6079 & 0.7515 & 0.7088 & 0.6074 & 0.5814 & 0.8860 & 0.6951 \\
\hline 0.6666 & 0.7011 & 0.6515 & 0.6665 & 0.6449 & 0.6235 & 0.7100 & 0.6912 & 0.8175 & 0.7395 \\
\hline 0.6594 & 0.6413 & 0.5298 & 0.6326 & 0.8004 & 0.7052 & 0.6988 & 0.6724 & 0.7996 & 0.8162 \\
\hline 0.7253 & 0.6290 & 0.7401 & 0.6842 & 0.7626 & 0.5908 & 0.7647 & 0.6459 & 0.6672 & 0.7639 \\
\hline 0.5708 & 0.1347 & 0.6696 & 0.8512 & 0.7659 & 0.7602 & 0.7624 & 0.7294 & 0.6208 & 0.7086 \\
\hline 0.0952 & 0.7362 & 0.7100 & 0.6619 & 0.7426 & 0.8276 & 0.7771 & 0.7003 & 0.7340 & \\
\hline
\end{tabular}

\section{CONCLUSIONS}

The index Cpmk indeed provides more quality assurance with respective to process yield and process loss to the consumers. In this paper the designing aspects of variable sampling plan based on process capability index $C_{p m k}$ for prescribed $\mathrm{C}_{\mathrm{AQL}}$ and $\mathrm{C}_{\mathrm{LTPD}}$ involving a minimum sum of risk are discussed. Table, which could be used for choosing the plan parameters for specified requirements, are constructed, and illustrations are provided.

\section{REFERENCES}

1. Aminzadeh, M. S. (1996), 'Inverse-Gaussian Acceptance Sampling Plans by Variables', Communications in Statistics - Theory and Methods, 25, pp. 923 - 935.

2. Aslam, M., Balamurali, S., Azam, M., Rao, G.S. \& Jun, C.H. 2015a. Mixed multiple dependent statesampling plans based on process capability index. Journal of Testing and Evaluation, 43(1):171-178.

3. Aslam, M., Khan, N. \& Khan, H. 2015b. SkSP-V Acceptance sampling plan based on process capabilityindex. Chiang Mai Journal of Science, 42(1):258-267.

4. Aslam, M., Wu, C.W., Azam, M. \& Jun C.H. 2013. Sampling inspection by variables for resubmittedlots based on process capability index Cpkfor normally distributed items. Applied MathematicalModeling, 37(3):667-675.

5. Aslam, M., Wu, C.W., Azam, M. \& Jun, C.H. 2014. Mixed acceptance sampling plans for productinspection using process capability index. Quality Engineering, 26(4):450-459. 
6. Bowker, A. H. and Goode, H. P. (1952), Sampling Inspection by Variables. McGraw-Hill, New York, Inc.

7. Das, N. G. and Mitra, S. K. (1964), 'The Effect of Non-Normality on Plans for Sampling Inspection by Variables',Sankhya - A, 26, pp.169 - 176.

8. Duncan, A. J. (1986), 'Quality Control and Industrial Statistics', Richard D. Irwin, Homewood, Illinois, USA..

9. Geetha, S., and Vijayaraghavan, R. (2010)'Procedure for Selection of Single Sampling Plans by Variables Based on Laplace Distribution'. Recent Trends in Statistical Research (A Publication of ManonmaniamSundaranar University, Tirunelveli, India), pp. $209-218$.

10. Geetha, S., and Vijayaraghavan, R. (2011b), 'A Procedure for Selection of Single Sampling Plans by Variables Based on Pareto Distribution'. Journal of Quality and Reliability Engineering, Vol. 2013, pp. 1 - 13

11. Geetha, S., and Vijayaraghavan, R. (2013)'Evaluation of Single Sampling Plans by Variables Based on Rayleigh Distribution'. Collection of Recent Statistical Methods and Applications (A Publication of Department of Statistics, University of Kerala, Trivandrum, India), pp. $315-324$

12. Golub, A. (1953): Designing Single-Sampling Inspection Plans when the Samplesize is Fixed. Journal of the American Statistical Association 48, 278-288.

13. Govindaraju, K and Subramani, K (1992): Selection of Double Sampling AttributesPlan for Given Acceptable Quality Level and Limiting Quality Level. Communicationsin Statistics - Simulation and Computation 21, 221-242.

14. Guenther, W. C. (1972), 'Variables Sampling Plans for the Poisson and the Binomial', StatisticaNeerlandica, 26, pp. 113 120.

15. Guenther, W. C. (1985), 'LQL Like Plans for Sampling by Variables', Journal of Quality Technology, 17, pp.155 - 157.

16. Hamaker, H. C. (1979), 'Acceptance Sampling for Percent Defective by Variables and by Attributes', Journal of Quality Technology, 11, pp. $139-148$.

17. Azarudheen, S., \& Pradeepaveerakumari, K. Design And Evaluation Of Quick Switching System With Single Sampling Plan As Reference Plan Under The Conditions Of Intervened Poisson Distribution.

18. Kaviyarasu V, Sangameshwaran.G,(2014) Designing of Quick Switching Multiple Deferred Sampling System - 3 through Minimum Angle and Minimum Sum of Risk

19. Kotz, S., Johnson, N.L., 2002. Process capability indices - A review, 1992-2000. Journal of Quality Technology 34 (1), 1-19.

20. Kotz, S., Lovelace, C., 1998. Process Capability Indices in Theory and Practice. Arnold, London, UK.

21. Lieberman, G. J. and Resnikoff, G. J. (1955), 'Sampling Plans for Inspection by Variables', Journal of the American Statistical Association, 50, pp. 457 - 516.

22. Montgomery, D. C. (2004), 'Introduction to Statistical Quality Control', John Wiley \& Sons, Inc., New York, USA.

23. Negrin, I., Parmet, Y. \&Schechtman, E. (2009). Developing a sampling plan based on Cpk. Quality Engineering, 21:306-318.

24. Negrin, I., Parmet, Y. \&Schechtman, E. (2011). Developing a sampling plan based on Cpk-

25. unknown variance. Quality and Reliability Engineering International, 27:3-14.

26. Owen, D. B. (1966), 'One-Sided Variables Sampling Plans', Industrial Quality Control, 22, pp. 450 - 456.

27. Owen, D. B. (1967), 'Variables Sampling Plans Based on the Normal Distribution', Technometrics, 9, pp. 417 - 423. 
28. Owen, D. B. (1969), 'Summary of Recent Work on Variables Acceptance Sampling with Emphasis on Non-Normality', Technometrics, 11, pp. $631-637$.

29. Pearn, W.L., Kotz, S., Johnson, N.L., 1992. Distributional and inferential properties of process capability indices. Journal of QualityTechnology 24 (4), 216-233.

30. Pearn, W.L., Lin, G.H., Chen, K.S., 1998. Distributional and inferential properties of the process accuracy and process precision indices. Communications in Statistics: Theory and Methods 27 (4), 985-1000.

31. Schilling, E. G. (1982), 'Acceptance Sampling in Quality Control', Marcel Dekker, New York, USA..

32. Senthilkumar D, LokanayakiK,EshaRaffie B(2014) Construction and Selection of Multiple

33. Deferred State (MDS-1) Variables SamplingPlan Involving Minimum Sum of Risks, Vol. 3, Issue 7,

34. Srivastava, A. B. L. (1961), 'Variables Sampling Inspection for Non-Normal Samples', Journal of Science and Engineering Research, 5, pp. 145 - 152.

35. Hadawale, K., \& Barve, S. (2013). Routing schemes in cognitive radio network. International Journal of Mathematics and Computer Applications Research (IJMCAR) ISSN, 2249-6955.

36. Soundararajan V. (1981): “Single Sampling Attributes Plan Indexed by AQL and AOQL”, Journal of Quality Technology, Vol.13, pp.195-200.

37. Soundararajan, V. and Vijayaraghavan, R. “Construction and Selection of Multiple Deferred State Sampling Plan”, Journal of Applied Statistics, Vol.17, No.3, pp.397-409, 1990.

38. Subramani, K., \&Govindaraju, K. (1990). Selection of multiple deferred state MDS-1 sampling plan for given acceptable and limiting quality levels involving minimum risks. Journal of Applied Statistics, 17(3), 431-434.

39. Subramani,K.,(2004) Minimum Risk Acceptance Sampling Plans:A Review Economic Quality Control Vol 19 (2004), No. 1, $121-126$

40. Suresh, R. P., Ramanathan, T. V. (1997, 'Acceptance Sampling Plans by Variables for a Class of Symmetric Distributions', Communications in Statistics - Simulation and Computation, 26, pp.1379 - 1391.

41. Takagi, K. (1972), 'On Designing Unknown-Sigma Sampling Plans based on a Wide Class of Non-Normal Distributions', Technometrics, 14, pp. 669 - 678.

42. Wu, C.W., Pearn, W.L., (2008) A variables sampling plan based on Cpmk for product

43. acceptance determination, European Journal of Operational Research 184 549-560

44. Zimmer, W. J. and Burr, I. W. (1963), 'Variables Sampling Plans based on Non-Normal Populations', Industrial Quality Control, 21, pp. 18 - 26. 
